\title{
Bias reduction for low-statistics PET: Maximum likelihood reconstruction with a modified Poisson distribution
}

\author{
Katrien Van Slambrouck, Simon Stute, Claude Comtat, Merence Sibomana, Floris H.P. van Velden, Ronald \\ Boellaard and Johan Nuyts
}

\begin{abstract}
Positron emission tomography (PET) data are typically reconstructed with maximum likelihood expectation maximization (MLEM). However, this method suffers from positive bias due to the non-negativity constraint. This is particularly problematic for tracer kinetic modeling analysis of dynamic PET studies.

Two reconstruction methods with bias reduction properties that do not use strict Poisson optimization are presented and studied in this work. They are compared to each other, to filtered backprojection (FBP), and to MLEM.

The first method is an extension of the so-called NEGML algorithm. This algorithm is based on a modified Poisson distribution that replaces the original function by a Gaussian distribution for low count data points. This modified likelihood function is optimized by a gradient ascent approach. The point of transition between the Gaussian and the Poisson regime is a parameter of the model.
\end{abstract}

The second method, AML, is a simplification of the ABMLmethod proposed by Byrne. ABML has parameters $A$ and $B$, which represent the lower and upper bounds for the reconstructed image. AML is the ABML algorithm with upper bound $B$ set to infinity. AML with negative $A$ has bias reduction properties. AML for different choices of $A$ is studied.

The parameter of both algorithms determines the effectiveness of the bias reduction. It was found that the parameter should be chosen large enough to ensure bias-free images. This means that both algorithms become more similar to a least squares algorithm, which turned out to be necessary to obtain bias-free reconstructions. This comes at the cost of increased variance. Nevertheless, both NEGML and AML have lower variance compared to FBP.

Furthermore, it was observed that the way randoms are handled has a large influence on the bias in the images. Reconstruction with smoothed randoms results in lower bias compared to reconstruction with unsmoothed randoms or randoms precorrected data. However, NEGML and AML results are both bias-free for large values of their parameter.

\section{INTRODUCTION}

Nowadays positron emission tomography (PET) reconstruction is mainly done by applying iterative reconstruction meth-

Katrien Van Slambrouck and Johan Nuyts are with the Department of Imaging and Pathology: Division of Nuclear Medicine and Medical Imaging Research Center, K.U.Leuven, B-3000 Leuven, Belgium. Simon Stute and Claude Comtat are with the CEA, DSV, I2BM, SHFJ, Orsay, F-91401, France. Merence Sibomana is with Sibomana Consulting SPRL, Emines, Belgium. Floris H.P. van Velden and Ronald Boellaard are with the Department of Radiology and Nuclear Medicine, VU University Medical Center, PO box 7057, 1007MB Amsterdam, The Netherlands. Corresponding author: johan.nuyts@uzleuven.be. Copyright (c) 2010 IEEE. Personal use of this material is permitted. However, permission to use this material for any other purposes must be obtained from the IEEE by sending a request to pubspermissions@ieee.org. ods. Iterative reconstruction is based on a forward model, which offers the possibility to model the true acquisition process better than analytical methods, e.g. by incorporation of finite resolution, irregularities in the geometry, etc. Iterative reconstruction in PET is usually based on a maximum likelihood (ML) approach, to take into account the Poisson statistics of the measured data. To suppress the noise propagation, the likelihood can be combined with a prior that favors smooth reconstructions [1].

The most popular iterative ML method for PET reconstruction is ML expectation maximization (MLEM [2], [3]). MLEM reconstructions tend to be biased in regions with low activity, in particular if these regions are surrounded by high activity structures. Moreover, MLEM suffers from noise induced bias [4]. This means that for kinetic PET studies, analytical methods, like filtered backprojection (FBP), are still the method of choice despite the fact that these images have often lower resolution and more streak artifacts due to noise [5], [6]. Dynamic PET data have often very limited numbers of counts that are sparsely divided over the lines of response. This is due to the fact that the early frames are often very short and for late frames only limited activity might be left due to the decay of the activity [6]. When bias is present in the derived time-activity curves, the resulting kinetic rate constants will also be biased. Increased variance is usually less problematic, because its influence is suppressed by fitting the kinetic model to a fairly large number of data points [11].

In every iteration of the MLEM algorithm, the current reconstruction image $\lambda$ is updated by adding the image $\Delta \lambda$ which is given for voxel $j$ by:

$$
\Delta \lambda_{j}=\frac{\lambda_{j}}{\sum_{i} c_{i j}} \sum_{i} c_{i j} \frac{y_{i}-\hat{y}_{i}}{\hat{y}_{i}}
$$

with

$$
\hat{y}_{i}=\sum_{j} c_{i j} \lambda_{j}+r_{i}
$$

where $y_{i}$ are the measured counts for detector pair $i, \hat{y}_{i}$ is the estimate of the sinogram mean based on the current reconstruction $\lambda, c_{i j}$ is the sensitivity of detector pair $i$ for activity in voxel $j$, and $r_{i}$ is the estimated number of scattered and/or random events.

The reconstruction formula in Eq. (1) shows that the update of a voxel is proportional to $\lambda_{j}$, the current activity estimate for voxel $j$. This means that regions with low activity with respect 
to the rest of the reconstruction, will converge much slower and a very high number of iterations is required to eliminate the positive bias. In practice one is not usually iterating long enough to avoid this incomplete convergence bias. Since there are other causes of bias, iterating longer by itself would not make the image bias-free.

The inherent non-negativity constraint in image space means that creating a region with zero mean is only possibly by making all voxels zero since no negative voxels values are allowed. Under noisy circumstances MLEM will always have some remaining positive bias in low-activity regions. FBP reconstruction has no constraints on the image values and has usually both positive and negative values in a cold region. In case of very noisy data (i.e. with very low counts), MLEM not only introduces bias in cold regions but also in regions with higher activity. It is known that MLEM with Poisson likelihood is only asymptotically unbiased, which means that it is only unbiased for an infinite number of counts [7], [8]. Each realization is forced to be positive and this positivity constraint is the origin of this bias. This property causes, even at convergence, positive bias in the image for low count measurements.

The ABML-method proposed by Byrne in [9] can be used for bias reduction. The ABML method extends MLEM by minimizing the Kullback-Leibler (KL) distance, between $y_{i}$ and $\hat{y}_{i}$, using a lower boundary $A$ and upper boundary $B$. It is based on a well chosen combination of KL distances [9] such that when applying the natural constraints of MLEM, $A=0$ and $B=\infty$, minimizing the KL cost function is equivalent to maximizing the Poisson likelihood. By setting $A$ to negative values, negative values in the image and sinogram domain are allowed, resulting in bias reduction behavior [10], [11], [12]. The convergence of different regions is still dependent on the activity but to a lesser extent. Since reconstruction values are usually not known beforehand, upper bound $B$ is often chosen very high and $A$ is often set to a very low value (i.e. a negative value with high magnitude). It was not evaluated whether less extreme values for $A$ would have an influence on bias reduction or convergence.

In [13], Nuyts et al. proposed the NEGML algorithm. It was originally developed to obtain images with a higher diagnostic value for reconstruction without attenuation correction. NEGML allows for negative values in the image domain. Moreover, the convergence of the different image parts was not dependent on $\lambda_{j}$ but a uniform weight was used for all voxels. Because of these two characteristics the algorithm could also be used to reduce bias, especially in cold regions surrounded by warm regions [14]. It was less successful for reconstruction based on very low count data, under those circumstances bias could still be observed. Moreover, its effectiveness seemed to be dependent on the implementation. The NEGML algorithm has a safety value that prevents division by zero and negative values. In [13] it was proposed to apply a lower limit of 1 on the denominator of the update formula. In other implementations a much smaller value was used, apparently leading to different bias reduction capacities [11].

In this work the NEGML algorithm is extended such that negative values in the image and in the sinogram are allowed.
This is obtained by replacing the Poisson distribution by a Gaussian distribution for small sinogram values. The bias reduction properties of this new NEGML algorithm and the influence of the transition point thereon will be evaluated in this work. A simplified version of ABML which is called AML is presented. In AML upper boundary $B$ is set to infinity. The influence of lower boundary $A$ on the bias is investigated. NEGML and AML will be compared to each other, to FBP, and to MLEM.

\section{Methods}

\section{A. NEGML}

Since the Poisson distribution is in theory the correct distribution, we prefer to use it whenever the number of counts is large enough to avoid introduction of bias and switch to another distribution when the number of counts is small. The determination of an optimal transition point between both distributions is part of the scope of this work.

The most obvious choice for a distribution which is close to Poisson and allows for negative values is a Gaussian distribution. Ignoring constant terms, the original Poisson loglikelihood as a function of the activity equals:

$$
\begin{aligned}
L(\hat{y}, y) & =\sum_{i} L_{i}\left(\hat{y}_{i}, y_{i}\right) \\
& =\sum_{i} y_{i} \ln \hat{y}_{i}-\hat{y}_{i} .
\end{aligned}
$$

Extended with a Gaussian part, the newly proposed likelihood becomes:

$$
L^{\psi}(\hat{y}, y)=\sum_{i} L_{i}^{\psi}\left(\hat{y}_{i}, y_{i}\right)
$$

with

$$
\begin{array}{lll}
L_{i}^{\psi}\left(\hat{y}_{i}, y_{i}\right) & \\
=y_{i} \ln \hat{y}_{i}-\hat{y}_{i} & \text { when } \quad \hat{y}_{i} \geq \psi \\
=-\frac{\left(y_{i}-\hat{y}_{i}\right)^{2}}{2 \psi}+y_{i} \ln \psi-\psi+\frac{\left(y_{i}-\psi\right)^{2}}{2 \psi} & \text { when } & \hat{y}_{i}<\psi
\end{array}
$$

where $\psi$ defines the point where the Poisson distribution switches to a Gaussian distribution. The last three terms in Eq. (6) ensure that the transition is continuous. Note that a Gaussian with a constant variance has been chosen. Figure 1 plots the original and new likelihood with $\psi=4$ for different values of $y_{i}$.

Computing the reconstruction $\lambda$ by maximizing the loglikelihood of eq. (6) is not straightforward. Therefore in every iteration $n+1$, the objective function $L^{\psi}(\hat{y} ; y)$ is approximated 


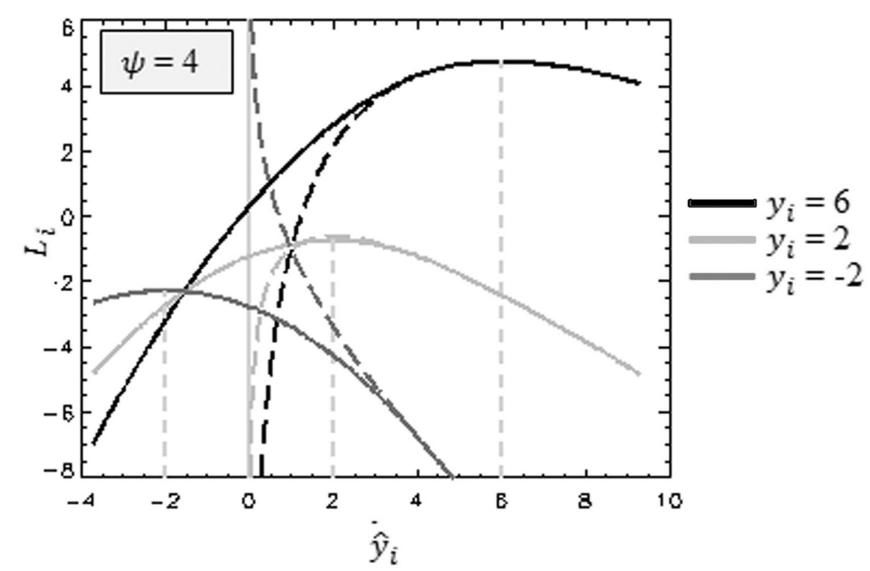

Fig. 1. The standard likelihood function (dashed lines) is drawn for $y_{i}=6$ (black), $y_{i}=2$ (light grey) and $y_{i}=-2$ (dark grey). The corresponding modified likelihood functions with $\psi=4$, as proposed in Eq. (6), are drawn as solid lines.

by the quadratic function $T^{\psi}\left(\hat{y} ; \hat{y}^{(n)}, y\right)$ as follows:

$$
\begin{aligned}
L^{\psi}(\hat{y} ; y) \simeq & T^{\psi}\left(\hat{y} ; \hat{y}^{(n)}, y\right) \\
= & \sum_{i} T_{i}^{\psi}\left(\hat{y}_{i} ; \hat{y}_{i}^{(n)}, y_{i}\right) \\
= & \sum_{i}-\frac{\left(y_{i}-\hat{y}_{i}\right)^{2}}{2 \max \left(\psi, \hat{y}_{i}^{(n)}\right)} \\
& +L^{\psi}{ }_{i}\left(\hat{y}_{i}^{(n)} ; y_{i}\right)+\frac{\left(y_{i}-\hat{y}_{i}^{(n)}\right)^{2}}{2 \max \left(\psi, \hat{y}_{i}^{(n)}\right)} \\
= & \sum_{i}-\frac{\left(y_{i}-\hat{y}_{i}\right)^{2}}{2 \max \left(\psi, \hat{y}_{i}^{(n)}\right)}+D_{i}
\end{aligned}
$$

where $D_{i}$ is independent of $\hat{y}_{i}$, and $\hat{y}^{(n)}$ denotes the calculated sinogram, obtained by applying (2) to the image $\lambda^{(n)}$ produced in iteration $n$. This function $T^{\psi}$ is equal to the likelihood $L^{\psi}$ in the current reconstruction and so are their first derivatives. Moreover, the derivatives of $L^{\psi}$ and $T^{\psi}$ have the same sign everywhere. Because they are both concave functions, this implies they have the same unique maximum.

$$
\begin{aligned}
L^{\psi}\left(\hat{y}^{(n)} ; y\right) & =T^{\psi}\left(\hat{y}^{(n)} ; \hat{y}^{(n)}, y\right) \\
\left.\frac{\partial L^{\psi}(\hat{y} ; y)}{\partial \hat{y}_{i}}\right|_{\hat{y}=\hat{y}^{(n)}} & =\left.\frac{\partial T^{\psi}\left(\hat{y} ; \hat{y}^{(n)}, y\right)}{\partial \hat{y}_{i}}\right|_{\hat{y}=\hat{y}^{(n)}} \\
\forall \hat{y}: \operatorname{sign}\left(\frac{\partial L^{\psi}(\hat{y} ; y)}{\partial \hat{y}_{i}}\right) & =\operatorname{sign}\left(\frac{\partial T^{\psi}\left(\hat{y} ; \hat{y}^{(n)}, y\right)}{\partial \hat{y}_{i}}\right)
\end{aligned}
$$

Using (2), $T^{\psi}\left(\hat{y} ; \hat{y}^{(n)}, y\right)$ can be rewritten as a function of the new reconstruction $\lambda$. However, it is convenient to rewrite this new reconstruction as an update of the result from the previous iteration as follows:

$$
\lambda_{j}^{(n+1)}=\lambda_{j}^{(n)}+\alpha_{j} \Delta x_{j},
$$

where we introduced a set of $\alpha_{j} \geq 0$ as design parameters. Now $T^{\psi}$ can be rewritten as a function of $\Delta x_{j}$ :

$$
T^{\psi}\left(\hat{y} ; \hat{y}^{(n)}, y\right)=T_{1}\left(\Delta x ; \lambda^{(n)}, y, \alpha\right),
$$

To optimize $T_{1}$, a gradient ascent algorithm as described in [13], [15], is applied. First, a series expansion of $T_{1}$ around $\Delta x=0$ is computed, applying the chain rule and noting that $\partial \hat{y}_{i} / \partial \Delta x_{j}=\alpha_{j} c_{i j}$ is a constant (see eq. (2)):

$$
\begin{aligned}
& T_{1}\left(\Delta x ; \lambda^{(n)}, y, \alpha\right) \\
& =T^{\psi}\left(0 ; \lambda^{(n)}, y\right) \\
& +\sum_{j} \sum_{i} \frac{\partial T^{\psi}}{\partial \hat{y}_{i}} c_{i j} \alpha_{j} \Delta x_{j} \\
& +\frac{1}{2} \sum_{j, k} \sum_{i} \frac{\partial^{2} T^{\psi}}{\partial \hat{y}_{i}^{2}} c_{i j} c_{i k} \alpha_{j} \alpha_{k} \Delta x_{j} \Delta x_{k},
\end{aligned}
$$

where the derivatives are evaluated in $\hat{y}=\hat{y}^{(n)}$. Since $T_{1}$ is a quadratic function, the second order expansion is exact. The second derivative of $T^{\psi}$ is always negative:

$$
\frac{\partial^{2} T^{\psi}}{\partial \hat{y}_{i}^{2}}=-\frac{1}{\max \left(\psi, \hat{y}^{(n)}\right)} \text {. }
$$

Using in addition the inequality $2 \Delta x_{j} \Delta x_{k} \leq\left(\Delta x_{j}\right)^{2}+$ $\left(\Delta x_{k}\right)^{2}$, a surrogate function $T_{2}$ for $T_{1}$ can be defined. This surrogate function is equal to $T_{1}$ in the current reconstruction $\lambda^{(n)}$ and lies below $T_{1}$ elsewhere:

$$
\begin{aligned}
& \left.T_{1}(D(\alpha) \Delta x, \lambda, y) \geq T_{2}(D(\alpha) \Delta x, \lambda, y)\right) \\
& =L^{\psi}\left(\hat{y}^{n}, y\right)+\sum_{j} \sum_{i} \frac{\partial T^{\psi}}{\partial \hat{y}_{i}} c_{i j} \alpha_{j} \Delta x_{j} \\
& +\frac{1}{2} \sum_{j, k} \sum_{i} \frac{\partial^{2} T^{\psi}}{\partial \hat{y}_{i}^{2}} c_{i j} c_{i k} \alpha_{j} \alpha_{k}\left(\Delta x_{j}\right)^{2}
\end{aligned}
$$

Maximization of $T_{2}$ is guaranteed to increase $T_{1}$. Because every term of $T_{2}$ depends on a single $\Delta x_{j}$ only, maximization of $T_{2}$ is straightforward and yields:

$$
\begin{aligned}
\Delta x_{j}^{(n+1)} & =\underset{\Delta x_{j}}{\operatorname{argmax}} T_{2}\left(\Delta x ; \lambda^{(n)}, y, \alpha\right) \\
& =-\frac{\sum_{i} c_{i j} \frac{\partial T^{\psi}}{\partial \hat{y}_{i}}}{\sum_{i} c_{i j} \frac{\partial^{2} T^{2}}{\partial \hat{y}_{i}^{2}} \sum_{k} c_{i k} \alpha_{k}} .
\end{aligned}
$$

Using Eq. (11) and expanding the derivatives produces the new version of the NEGML algorithm:

$$
\lambda_{j}^{(n+1)}=\lambda^{(n)}+\frac{\alpha_{j} \sum_{i} c_{i j} \frac{y_{i}-\hat{y}_{i}}{\max \left(\psi, \hat{y}_{i}^{(n)}\right)}}{\sum_{i} c_{i j} \frac{\sum_{k} c_{i k} \alpha_{k}}{\max \left(\psi, \hat{y}_{i}^{(n)}\right)}} .
$$

The original NEGML algorithm [13] is obtained when setting $\psi=1$ and $\alpha_{j}=1$. Neither $\psi$ nor $\alpha_{j}$ were explicit parameters in the original NEGML work. Instead of using $\psi$, division by zero was avoided by restricting the denominator to values larger than or equal to 1 . Since $\hat{y}_{i}$ is a number of photons, $\hat{y}_{i} \geq 1$ was chosen as a reasonable value and gave good results. In other implementations of NEGML, this restriction was sometimes set at much smaller values e.g. $\hat{y}_{i} \geq 10^{-4}$ [11]. The results of different implementations were different regarding bias reduction, probably due to the different restriction for the denominator. The experiments in this work will explicitly test the influence of $\psi$ on convergence and bias values.

When only using the Poisson likelihood, i.e. $\hat{y}_{i} \geq \psi$ for all $i$, and by using $\alpha_{j}=\lambda_{j}^{(n)}$ and $r_{i}=0$, the update becomes the MLEM update. The original NEGML algorithm used a 
mixed update step to improve the convergence in high activity regions. The update obtained for MLEM and NEGML were both calculated for voxel $j$ and the largest of both was applied. This mixed update is equivalent to over-relaxation and might cause convergence problems. Hence, we prefer to use a pure NEGML update.

The convergence of high activity regions could be improved by altering the values for $\alpha_{j}$. This parameter was introduced in our work on metal artifact reduction and iterative reconstruction in CT [15], [16] and serves as a voxel convergence weight during reconstruction. Similar weights were used in the grouped coordinate algorithm by Fessler et al. [17]. Choosing $\alpha_{j}=\lambda_{j}^{(n)}$ gives the weighting used in MLEM, choosing $\alpha_{j}=1$ results in the weighting of the original NEGML algorithm. A compromise could be obtained by defining weights that have a weaker dependence on the activity but still assign higher weights to high activity regions.

NEGML can be accelerated with ordered subsets, similarly as proposed for MLEM by Hudson and Larkin in [18].

\section{B. $A M L$}

The ABML algorithm presented by C. Byrne [9] allows to perform an MLEM-like reconstruction between an image upper bound $B$ and lower bound $A$ by optimizing a well chosen combination of KL distances (see [9]). This KL cost function can be considered as the sum of KL distances between $y_{i}$ and $\hat{y}_{i}$, using $A$ and $B$ as offsets. ABML can be seen as an extension of the MLEM algorithm, because when using the natural boundaries for MLEM, $A=0$ and $B=\infty$, the KL cost function is equal to the Poisson likelihood and ABML becomes equivalent to MLEM.

The ABML reconstruction formula as used by Erlandsson et al. in [10] is:

$$
\lambda_{j}^{\text {new }}=\frac{B_{j} P_{j}+A_{j} Q_{j}}{P_{j}+Q_{j}}
$$

with

$$
\begin{aligned}
P_{j} & =\frac{\left(\lambda_{j}-A_{j}\right)}{\sum_{i} c_{i j}} \sum_{i} c_{i j} \frac{y_{i}-\sum_{k} c_{i k} A_{k}}{\hat{y}_{i}-\sum_{k} c_{i k} A_{k}} \\
Q_{j} & =\frac{\left(B_{j}-\lambda_{j}\right)}{\sum_{i} c_{i j}} \sum_{i} c_{i j} \frac{\sum_{k} c_{i k} B_{k}-y_{i}}{\sum_{k} c_{i k} B_{k}-\hat{y}_{i}}
\end{aligned}
$$

ABML can be used as a bias reduction algorithm by setting $A_{j}$ to negative values. This way it allows for negative values in the image and the sinogram domain. It has been shown to reduce bias substantially [10], [11]. Often a single value for $A_{j}$ is chosen, i.e. $A_{j}=A$ for all voxels. This sets the lower limit for the reconstruction values to $A$ and for the sinogram values to $A \sum_{k} c_{i k}$. Since there is no reason to have an upper bound for the image, $B_{j}$ is usually set to a value much larger than the expected maximum activity. Introducing some approximations ABML can be extended to an ordered subsets version [10] with inclusion of randoms [11].

Since very large values are usually chosen for $B$, we propose to use ABML with infinite upper bound (as in MLEM), referred to as AML. This simplifies the expressions and avoids numerical problems that might occur for extreme values of $B$ in Eq. (19). The AML update step for $\lambda_{j}$, with uniform $A$, in additive form becomes:

$$
\Delta \lambda_{j}=\frac{\lambda_{j}-A}{\sum_{i} c_{i j}}\left(\sum_{i} c_{i j} \frac{y_{i}-\hat{y}_{i}}{\hat{y}_{i}-A \sum_{k} c_{i k}}\right) .
$$

This update step can be considered as an update where the image and sinogram are shifted, with $A$ and $A \sum_{k} c_{i k}$ respectively, for calculating the update step. This means that the Poisson distribution is evaluated at higher values where the influence of the non-negativity constraint is negligible. Before adding this to the current reconstruction the update is shifted back. Note that choosing $A=0$ results in the original MLEM algorithm. The factor $\left(\lambda_{j}-A\right)$ in Eq. (20) still represents an activity dependent weight for the update of voxel $j$, however, the weights become more uniform when $A$ becomes more negative. Hong et al [19] developed a variant of ABML that also allows for negative image values and reduces the influence of the non-negativity constraint during reconstruction by combining multiple time frames.

The parameter $A$ is defined in image space. It is not straightforward to determine a lower bound such that biasfree images are ensured (i.e. such that negative values are not suppressed). To our knowledge no guidelines for choosing such a value for $A$ have been published. When the lower bound is not low enough, bias might still occur. Therefore, $A$ is usually set to a relatively extreme (negative) value to make sure that any constraint on the negativity has been eliminated. As argued in the next section, this turns the algorithm effectively into a least squares algorithm. It is unclear if such an extreme value is the best choice.

\section{Unweighted least squares and extreme values for $\psi$ and $A$}

The influence of the parameter $\psi$ and $A$ on the reconstructions of NEGML and AML will be studied in this paper. The behavior of NEGML and AML for extremely high values for $\psi$ and extremely low values of $A$ will be studied as well. For large $\psi$, all sinogram pixels will be in the Gaussian regime. It is therefore expected that NEGML will become more and more similar to unweighted least squares reconstruction. The same holds for AML. When $|A|$ is large $^{1}$, the shift from $A$ will be so large that the difference in Poisson weighting will be very small, eliminating all weighting difference in practice.

The NEGML algorithm for $\alpha_{j}=1$ with $\psi \rightarrow \infty$ becomes:

$$
\Delta \lambda_{j}=\frac{\sum_{i} c_{i j}\left(y_{i}-\hat{y}_{i}\right)}{\sum_{i} c_{i j}\left(\sum_{k} c_{i k}\right)} .
$$

This update formula is used whenever $\hat{y}_{i} \leq \psi$ for all $\hat{y}_{i}$.

The AML algorithm with $A \rightarrow-\infty$ yields:

$$
\Delta \lambda_{j}=\frac{1}{\sum_{i} c_{i j}} \sum_{i} c_{i j} \frac{\left(y_{i}-\hat{y}_{i}\right)}{\sum_{k} c_{i k}} .
$$

Unlike for NEGML this formula is theoretically only valid when $A=-\infty$ since all finite values for $A$ will still follow the Poisson distribution at $\hat{y}_{i}-A \sum_{h} c_{i h}$. However, this

\footnotetext{
${ }^{1}$ To avoid confusion when discussing negative values of $A,|A|$ is used Since this paper only considers negative values of $A,|A|$ is unambiguous Large $|A|$ thus means a negative value of $A$ with large magnitude.
} 
distribution becomes very flat for large $|A|$. For comparison, the typical least squares algorithm has the following form:

$$
\Delta \lambda_{j}=\frac{\rho}{\sum_{i} c_{i j}} \sum_{i} c_{i j}\left(y_{i}-\hat{y}_{i}\right)
$$

with $\rho$ a relaxation factor. The results in Eq. (21) and (22) resemble the least squares update, but are not identical to it. Only when $\sum_{k} c_{i k}$ is constant, both algorithms yield the least squares expression.

\section{Additive contamination}

The additive contamination $r_{i}$ can be modeled as in Eq. (2). This is often referred to as ordinary Poisson, because it assumes that the randoms term $r_{i}$ is not noisy such that the Poisson model for $y_{i}$ is preserved. Ordinary Poisson is valid when an estimate of the randoms with low noise is available. This can be obtained by smoothing the randoms estimate [20] or by calculating them from the singles [21].

In some clinical PET systems, randoms precorrection is still applied. In this case, the randoms estimate is subtracted from the measurement, possibly leading to negative values in $y_{i}$. For reconstruction with MLEM, these values should be set to zero which already introduces bias in the data. FBP, NEGML and AML support negative sinogram values and no positivity requirement is needed. In [22] some modified statistical methods are given that specifically model randomsprecorrected PET emission data.

In the experiments described below, three different ways to correct for the randoms have been considered for the different reconstruction algorithms. For FBP, the randoms were always subtracted, with or without randoms smoothing. Since a uniform randoms contribution was used, this smoothing can be done with a Gaussian function. More dedicated smoothing algorithms have been developed for real PET systems [20]. For the iterative algorithms the following reconstructions can be used, where $s_{i}$ is the noisy estimate of the randoms and $s_{i}^{S}$ is a smoothed randoms estimate (full width half maximum $(\mathrm{FWHM})=5$ pixels $)$ :

- Randoms precorrection with randoms smoothing: $y_{i} \rightarrow$ $y_{i}-s_{i}^{S}$

- Ordinary Poisson with randoms smoothing: $y_{i}$ and $r_{i}=$ $s_{i}^{S}$

- Ordinary Poisson without randoms smoothing: $y_{i}$ and $r_{i}=s_{i}$

\section{EXPERIMENTS}

This section describes the experiments that are performed to evaluate the bias reduction capacity of NEGML and AML, compared to FBP and MLEM. The experiments are based on two-dimensional simulations and consider the influence of the parameter $\psi$ and $A$ on the bias, variance, convergence and noise characteristics of NEGML and AML, respectively, for two different phantoms simulated with different settings.

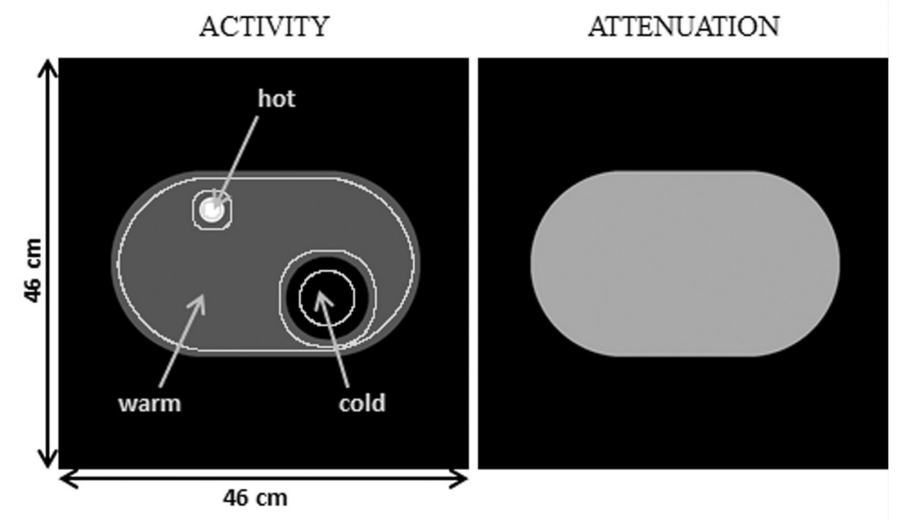

Fig. 2. Phantom 1. The phantom has a cold, warm and hot region. The activity for the main experiment was 0 for the cold region, 1 for the warm region and 4 for the hot region. In a second setting, the phantom's activity was multiplied by three. The phantom is assumed to have uniform water attenuation.

\section{A. Phantom 1}

1) Simulations: A two-dimensional phantom with a cold, warm and hot region, as shown in figure 2, is simulated. The activity in the image in activity per voxel is: 0 for the cold region, 1 for the warm region and 4 for the hot region. The phantom is discretized in an image of $46 \mathrm{~cm} \mathrm{x} 46 \mathrm{~cm}$. During simulation this image was represented by a $920 \mathrm{x}$ 920 pixel grid and a parallel beam simulation is performed with 920 lines of response per projection and 200 projections. Uniform water attenuation is applied. A uniform randoms contribution was simulated, assuming a Poisson distribution with the expectation equal to the mean of the uncontaminated sinogram. The system resolution was modeled by a Gaussian with FWHM of $5 \mathrm{~mm}$.

The obtained sinogram is rebinned to 230 lines of response per projection and 200 projections. This means that the simulation was four times oversampled. This simulation was repeated for 60 (Poisson) noise realizations and 50 time frames of increasing duration, resulting in data sets with mean count per sinogram pixel between 0.05 and 1000 counts. In a second simulation setting for this phantom, the activity in the image was multiplied by three.

2) Reconstructions: Reconstructions were performed with FBP, MLEM, NEGML and AML on a $230 \times 230$ pixel grid, with pixel size $2 \mathrm{~mm} \times 2 \mathrm{~mm}$. FBP was performed using a standard ramp filter. For NEGML different values of $\psi$ were evaluated: $\psi=\{1,4,9,16,25,100,100000\}, \alpha_{j}=1$ was used for all $j$. For AML different values of $A$ where used: $A=\{-1,-5,-10,-50,-100,-1000,-100000\}$. For all iterative methods 200 iterations were applied without the use of ordered subsets. During reconstruction the resolution was modeled by a Gaussian with FWHM $4 \mathrm{~mm}$, introducing a small mismatch with the resolution of the simulation.

The simulations were reconstructed with three different ways of randoms handling as listed in section II-D.

3) Evaluation: A cold, warm and hot region of interest (ROI) are defined in the phantom. The ROIs exclude all pixels close to the edges, as shown in figure 2. The bias is estimated 


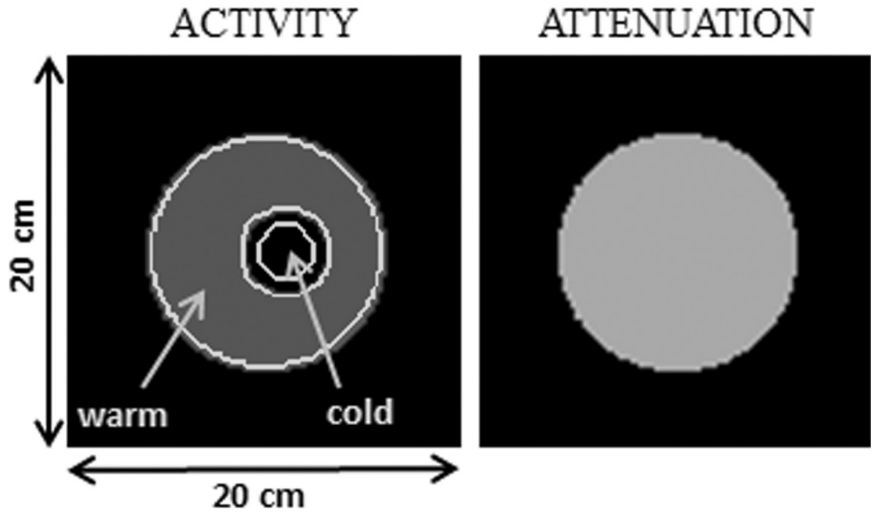

Fig. 3. Phantom 2. The phantom consists of a warm and cold region. The activity and attenuation are shown. The phantom is assumed to have uniform water attenuation.

by calculating the mean value in a ROI over the different noise realizations, the ROI mean, defined by:

$$
\text { ROI Mean }=\frac{1}{N J_{\mathrm{ROI}}} \sum_{n}^{N} \sum_{j \in \mathrm{ROI}} \lambda_{j, n}
$$

with $N$ the number of noise realizations and $J_{\mathrm{ROI}}$ the total number of pixels in the region. The ROI mean values are calculated for all 50 frames. The variance of the mean value over the noise realizations, the ROI Var, is calculated by:

$$
\text { ROI Var }=\frac{1}{N} \sum_{n}\left(\text { ROImean }-\frac{1}{J_{R O I}} \sum_{j \in \mathrm{ROI}} \lambda_{j, n}\right)^{2} .
$$

\section{B. Phantom 2}

This experiment is designed to further investigate whether the findings for phantom 1 and for the configuration described in the previous section, remain valid for other configurations. We will investigate whether changes in the number of projection lines (i.e. projection angles) for a fixed number of total counts has influence on the results. This will give an indication whether the results obtained in this study can be extended to fully 3D PET and time-of-flight PET, where the ratio of the number of sinogram pixels to the number of image voxels is much larger than in 2D PET. Because 3D (TOF) PET simulations are very time consuming, it is more practical to study this effect by simulating 2D PET with a much larger number of angles.

A different phantom with one cold and one warm region and three different projection settings are used for this experiment.

1) Simulations: A simple two-dimensional phantom consisting of a uniform disk with one cold region, depicted in figure 3 , is simulated. The activity in the warm region is set to 1 , in the cold region it is set to 0 . The phantom is discretized in an image of $20 \mathrm{~cm}$ x $20 \mathrm{~cm}$ using a 400x400 pixel grid.

To evaluate the dependence on the sinogram pixel values this phantom was simulated with three different settings. For setting 1, 100 projection angles were calculated, 500 for setting 2 and 1000 for setting 3 . However, the total number of counts for each simulation was kept constant, i.e., the sensitivity of each line of response of the simulated PET system was 5 times lower for setting 2 and 10 times lower for setting 3.

A parallel beam simulation with 400 lines of response per projection, was performed, assuming that the phantom was a uniform attenuator (consisting of water). A uniform randoms estimate was set equal to the mean of the uncontaminated sinogram. This simulated estimate was subjected to Poisson noise. The simulation was rebinned for reconstruction to 100 projection lines per angle.

For all three settings, 100 noise realizations were performed for 20 time frames of increasing duration. Only relatively short time frames were considered with mean sinogram count between 0.05 and 10 counts per pixel.

The system resolution was again modeled by a Gaussian with FWHM of $5 \mathrm{~mm}$.

2) Reconstruction: The simulated measurements were reconstructed on a $100 \times 100$ pixel grid (2 mm isotropic pixel size) with FBP, MLEM, NEGML $(\psi=\{1,4,16\}$, again with $\alpha_{j}=1$ for all $\left.j\right)$ and AML $(A=\{-1,-5,-50\})$. Precorrection of the randoms, using smoothed randoms (FWHM = 5 pixels), was used for FBP, Ordinary Poisson with smoothed randoms (FWHM $=5$ pixels) was applied for the iterative methods. Iterative reconstruction was performed for 20 iterations with 10 subsets. As for phantom 1, a small mismatch with the resolution in the simulation was created by using by a Gaussian with FWHM $=4 \mathrm{~mm}$ during reconstruction.

3) Evaluation: A warm and a cold ROI are defined as shown in figure 3. The ROI mean is calculated as in Eq. (24). Note that although all three settings have the same total amount of activity in the image and the sinogram, the mean count per sinogram pixel is larger for settings with fewer projection views.

\section{RESUlts}

\section{A. Phantom 1}

This section describes the main results of this work for the phantom depicted in figure 2. The ROI means as functions of the frame durations are shown in figure 4 . The frame duration is represented by the mean number of sinogram counts in the frame. The standard error on the mean is given in figure 5 .

$$
\begin{aligned}
& \text { Error mean }= \\
& \frac{1}{\sqrt{N-1}} \sqrt{\frac{1}{N} \sum_{n}\left(\text { ROI mean }-\frac{1}{J_{R O I}} \sum_{j \in \mathrm{ROI}} \lambda_{j, n}\right)^{2}}
\end{aligned}
$$

The results for ordinary Poisson with and without smoothed randoms and for randoms precorrection are shown. The upper row of the figure evaluates different values for $\psi$ in NEGML, in comparison with FBP and MLEM. The lower row gives the evaluation of AML for different values of $A$.

Ordinary Poisson with randoms smoothing has the lowest bias for all methods. In general MLEM has the highest bias, especially in the cold and warm region. The bias of NEGML and AML is dependent on the model parameter. The positive bias in the cold region is higher when the data are noisier. Evaluating the bias in the cold region with respect to the expected value in the warm region, $40 \%$ to $60 \%$ bias is observed for low count data. The bias only drops below $10 \%$ at on average 10 counts per sinogram pixels. The bias in 
NEGML Precorrection without randoms smoothing

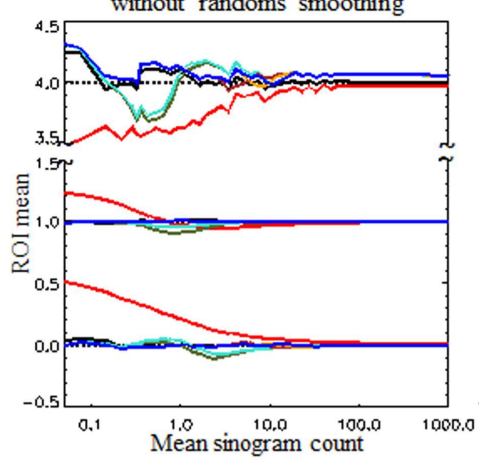

AML

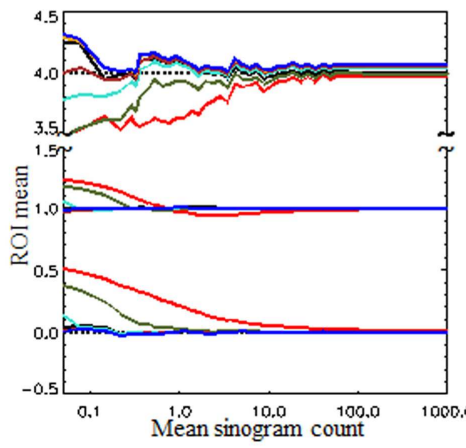

Ordinary Poisson with randoms smoothing
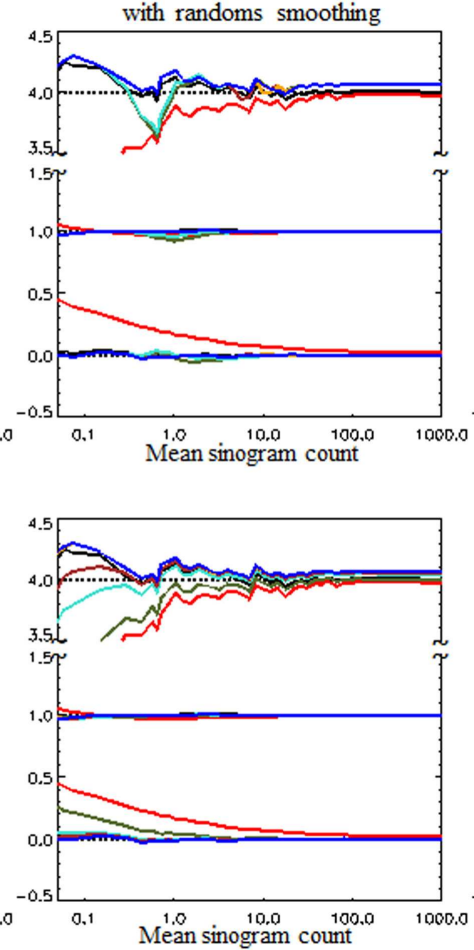

Ordinary Poisson without randoms smoothing

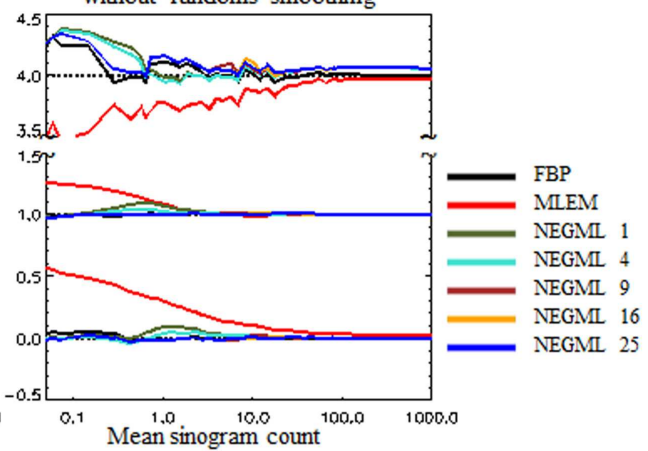

Fig. 4. Phantom 1. Evaluation of the ROI Mean for different frame durations. The frame duration is represented by the mean number of counts per sinogram pixel in the frame. Three different ways for randoms handling are shown. The upper row gives the results for NEGML, the lower row shows the results for AML.



Fig. 5. Phantom 1. The standard error on the mean for the Ordinary Poisson method with randoms smoothing (figure 4) for FBP, MLEM, NEGML 16 and AML -5 in the cold, warm and hot region of phantom 1 .

the warm region is less pronounced, lower than $10 \%$, for ordinary Poisson with randoms smoothing. It increases up to $40 \%$ for Ordinary Poisson without randoms smoothing and precorrection for very low count data. For NEGML all methods perform similarly with bias in the cold region mostly less than $2 \%$ (compared to the warm region). When the mean sinogram count has a value around $\psi$, a local increase in the bias can be observed. This is the most obvious in case of unsmoothed randoms. This local increase becomes less pronounced as $\psi$ increases. We have currently no good explanation for this behavior. AML is dependent on $A$ for bias reduction, the bias decreases with increasing $|A|$. This effect is again the most pronounced in case of unsmoothed randoms. For high values of $|A|$ the bias is also below $2 \%$ everywhere and increases when $|A|$ decreases.

In the hot region, even at relatively high counts, some remaining bias, of about $2 \%$, is observed for NEGML and for AML with large $|A|$. This bias reduces when the number of iterations increases (data not shown). For less noisy data, MLEM performs in general better than both NEGML and AML in the high activity region. Note that there seems to be positive bias in the high activity region for FBP. However, this is a random error, caused by the high amounts of noise and the limited number of noise realisations. We verified this with additional noise realisations for low counts (data not shown).

Figure 6 depicts the ROI Mean as a function of the frame duration for the cold region of the same phantom with threefold increased activity reconstructed with Ordinary Poisson with randoms smoothing. In comparison with the phantom with lower activity, AML has more bias, which increases for smaller $|A|$. The results for NEGML are very similar to the case with less activity in the image. The reconstructions remain almost bias free. The difference between AML and NEGML for increased activity is due to the fact that $A$ was not changed 
NEGML Ordinary Poisson

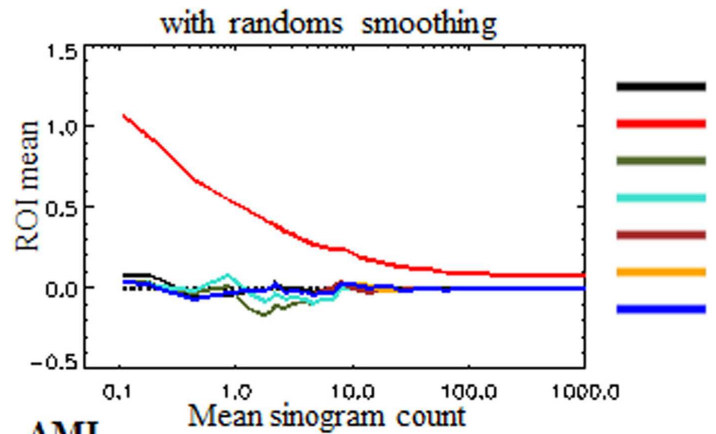

AML

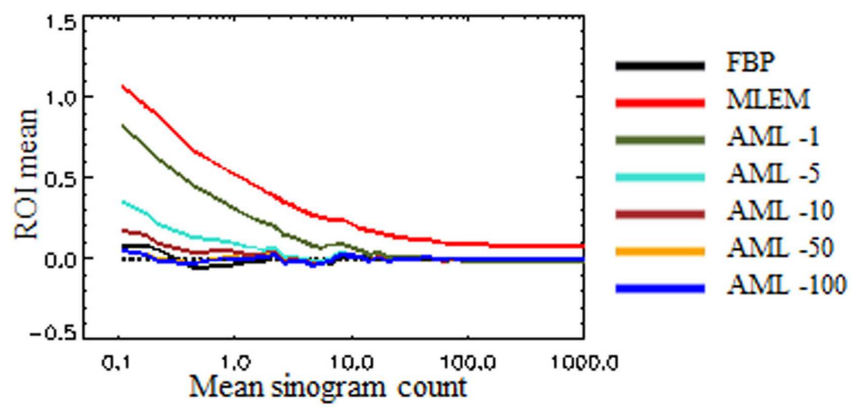

Fig. 6. Phantom 1 with threefold increased activity. Evaluation of the ROI Mean for the cold regions for different frame durations with ordinary Poisson. The frame duration is represented by the mean number of counts per sinogram pixel in the frame. The upper row gives the results for NEGML, the lower row shows the results for AML.

while the activity in the image did change. The influence of increased activity on the bias in the warm and hot regions was less pronounced (not shown).

The reconstructed images for the different methods with ordinary Poisson with randoms smoothing for on average 5 counts per sinogram pixel are shown in figure 7. After reconstruction, the images were smoothed with a Gaussian filter with FWHM of $4 \mathrm{~mm}$. The maximum number of counts in this sinogram is 19 with more than $95 \%$ of the sinogram pixels below 16 counts. The typical noise-induced streaks are the most pronounced in the FBP reconstruction. MLEM suffers the least from these streaks. NEGML and AML have only few streaks for small $\psi$ and small $|A|$. The streaks become more pronounced with increasing $\psi$ or increasing $|A|$. AML has pixel dependent convergence weighting which means that it has more MLEM-like characteristics. The hot region is therefore sharper in MLEM and AML with small $|A|$. With respect to noise streaks there is no obvious difference observed between bias-free NEGML and AML images, only that AML has somewhat more streaks in the background of the image.

The influence of the parameter $\psi$ or $A$ on the ROI Mean and ROI Var is shown in figure 8 . The frames represented in this figure have on average 1 or 5 counts per sinogram pixel. The variance in the cold and warm region is lower for NEGML and AML compared to FBP but still higher than for MLEM. For most parameter choices, the bias in NEGML and AML is better than for MLEM in the cold and warm regions. A local increase of the bias was observed when the mean sinogram count is close to the chosen value of $\psi$, the higher the value

\section{Ordinary Poisson with randoms smoothing}

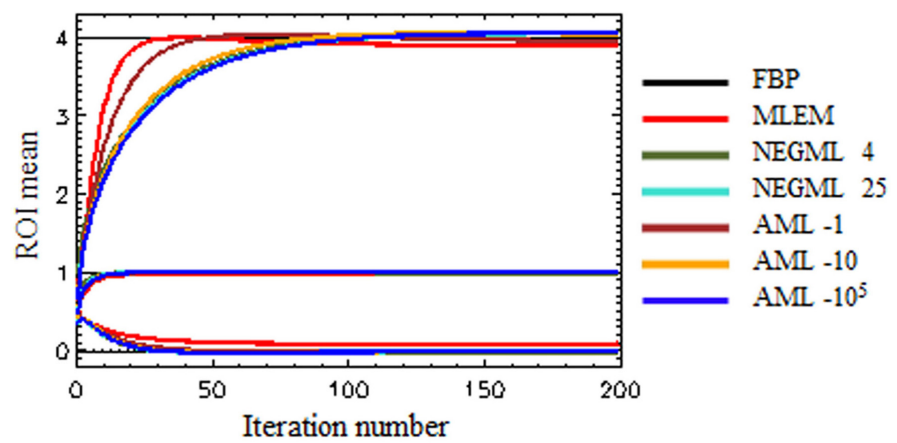

Fig. 9. Phantom 1. Convergence of MLEM, NEGML (with $\psi=\{4,25\}$ ) and AML (with $A=\left\{-1,-10,-10^{5}\right\}$ ). The value for FBP is given as a reference

of $\psi$ the smaller this increase in bias. This local increase is negligible from $\psi=16$ and larger. The bias due to incomplete convergence in the hot region, for NEGML and AML with large $|A|$, was expected from previous results.

The influence of $\psi$ and $A$ on the convergence of NEGML and AML for all three regions is given in figure 9, for a measurement with on average 5 counts per sinogram pixel. The result is shown for ordinary Poisson with randoms smoothing and for parameters: $\psi=\{4,25\}$ and $A=\left\{-1,-10,-10^{5}\right\}$. For this frame duration NEGML with $\psi=25$ worked completely in the Gaussian regime. The convergence for the cold and warm region is relatively fast. At 50 iterations all methods converged. In the hot regions MLEM and AML with small $|A|$ converge faster than NEGML and AML with larger $|A|$. An experiment with more iterations (not shown) indicates that most of the algorithms were not yet converged in the hot region, another 100 to 200 iterations are needed to obtain complete convergence and a hot region without bias.

\section{B. Phantom 2}

The graphs in figure 10 depict the ROI mean in the cold and warm region of phantom 2 for a set of short frame durations for FBP, MLEM, NEGML and AML. Three different projection setting, with a different number of projection angles were used.

FBP and NEGML show similar bias reduction for all three settings. For the smaller parameters, $\psi=\{1,4\}$, again a local increase is observed, this is the most obvious for the warm region. For $\psi=16$ no bias is observed for all three settings. The bias for MLEM and AML is not the same for all three settings. Although the activity in the image is the same for all settings, the same value for $A$ results in different bias reduction depending on the setting. The largest value of $|A|, A=-50$, results in bias-free reconstruction for all three settings.

\section{DISCUSSION}

Two methods for bias reduction have been presented. They both use a likelihood function which can be considered as a modified Poisson function.

NEGML uses a combination of a Poisson and Gaussian function with a transition between both functions at $\psi$ such 

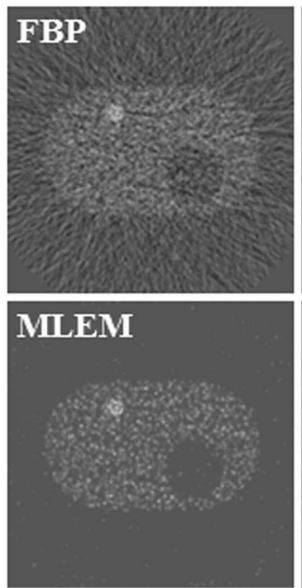
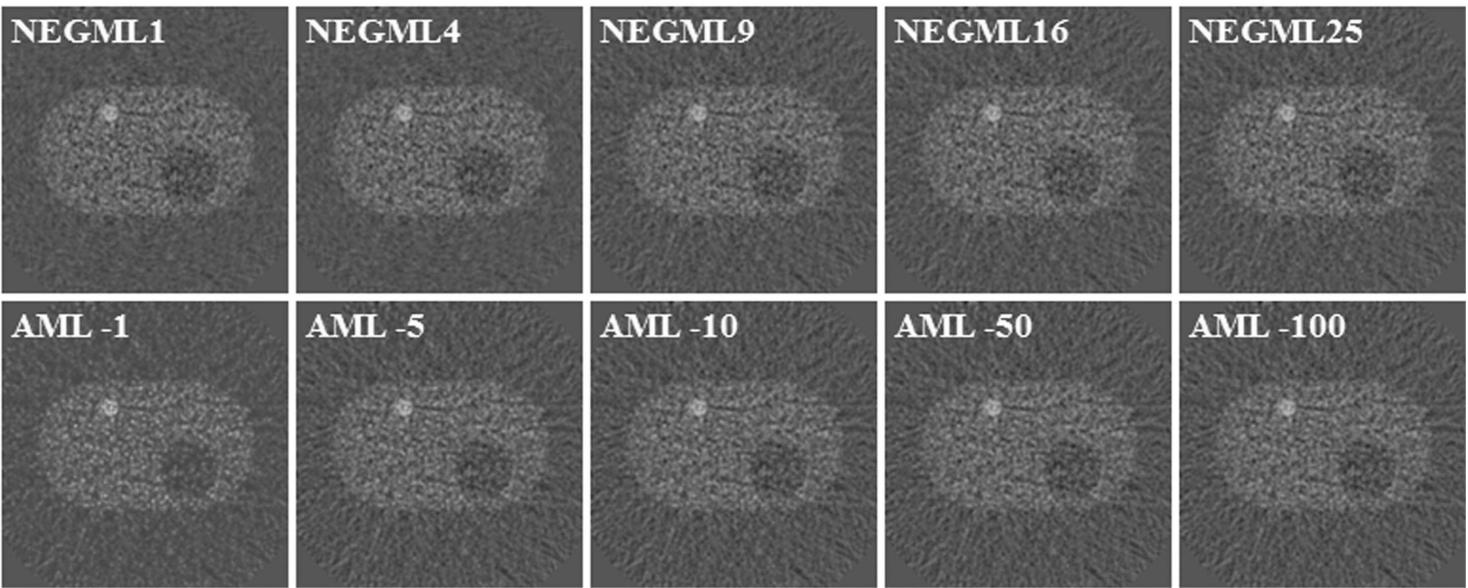

Fig. 7. Phantom 1. FBP, MLEM, NEGML $(\psi=\{1,4,9,16,25\})$ and AML reconstruction $(A=\{-1,-5,-10,-50,-100\})$. Ordinary Poisson with randoms smoothing.
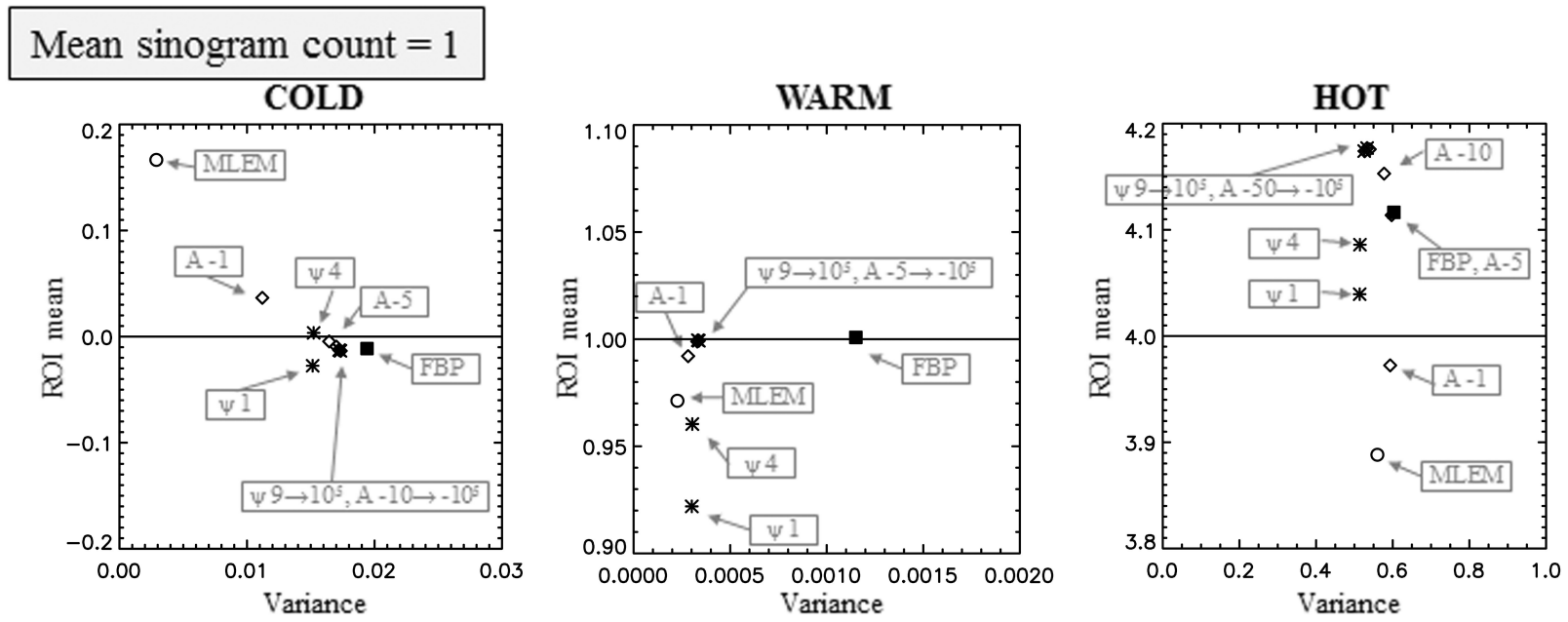

- FBP
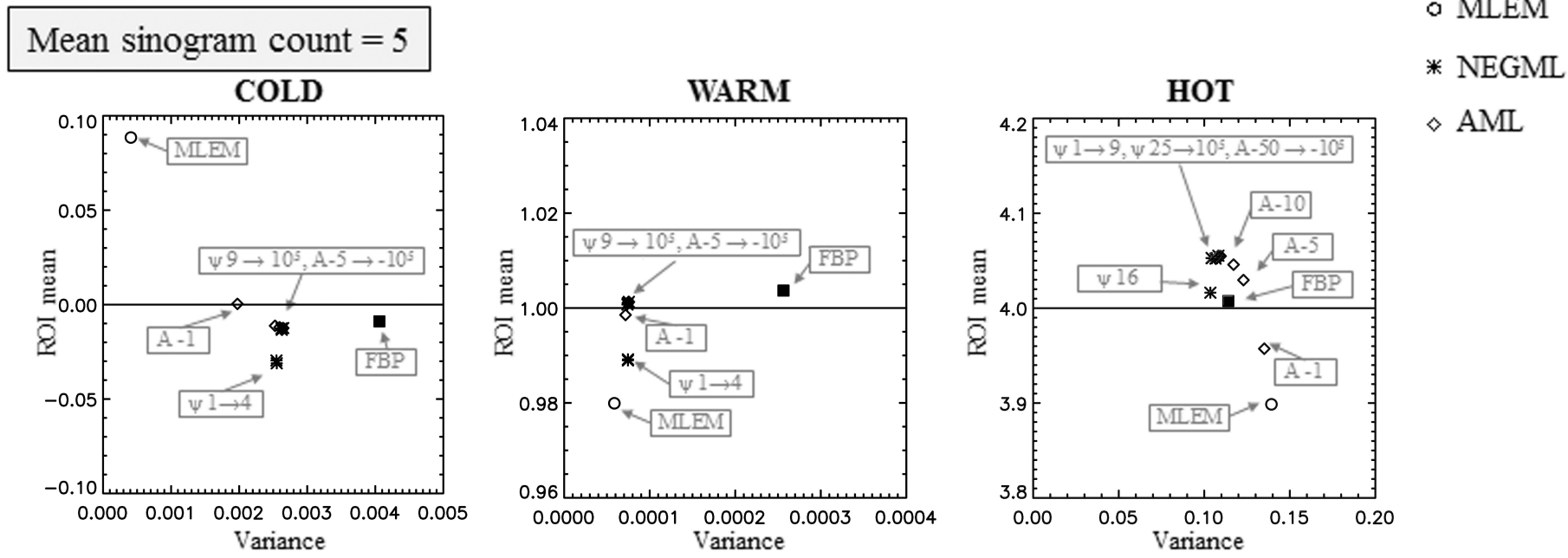

Fig. 8. Phantom 1. Mean with respect to variance in the cold regions, warm region and hot region for different values of $\psi$ and $A$. The chosen frame duration correspond to on average 1 and 5 counts per sinogram pixel. The solid line represent the true value for each region. Ordinary Poisson with randoms smoothing. 
Ordinary Poisson with randoms smoothing

FBP

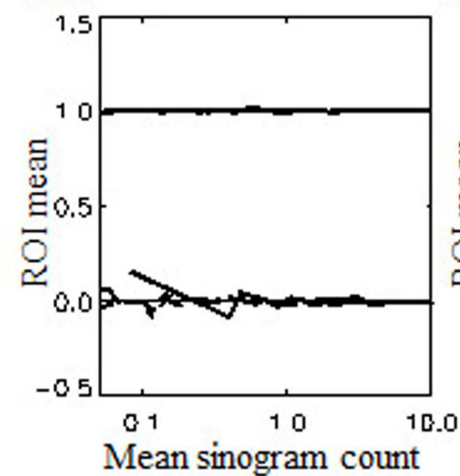

MLEM

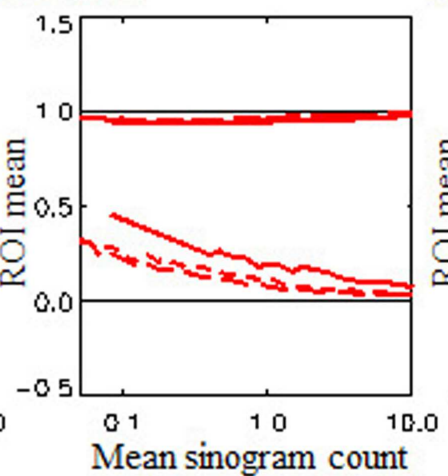

Setting 1 (100p)

Setting 2 (500p)

- Setting $3(1000 \mathrm{p})$
NEGML
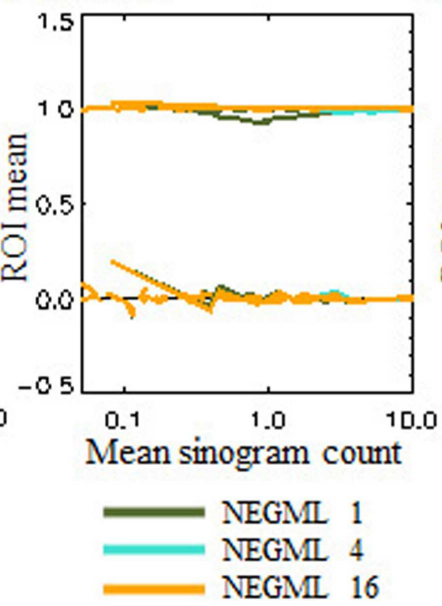

AML

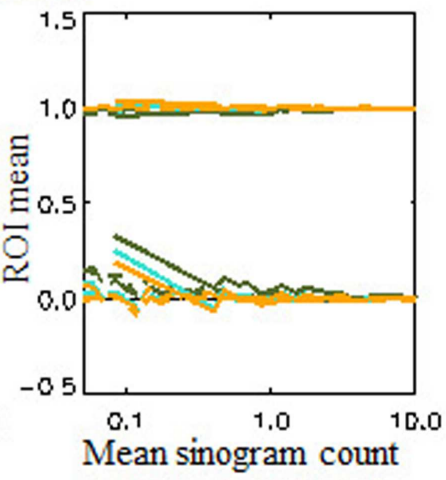

AMI -1

AML -5

AML -50

Fig. 10. Phantom 2. ROI mean in the cold and warm region for three setting. Setting 1: 100 projections angles (solid line), setting 2: 500 projection angles (small dashes), setting 3: 1000 projection angles (long dashes).

that negative sinogram values are allowed. AML is a modification of ABML with upper bound $B$ set to infinity, which simplifies and stabilizes (numerically) the algorithm. AML can be considered to apply a shift on the data such that they are evaluated at higher values where the influence of the positivity constraint is negligible.

The influence of the parameters $\psi$ and $A$ on the bias in the reconstructed images has been investigated. It was shown that both algorithms converge to least squares-like algorithms when their parameter is sufficiently large.

For NEGML, very low bias was observed for most values of $\psi$. For low values of $\psi$, a (so far unexplained) increase in bias was seen when the mean sinogram count was similar to $\psi$. Consequently, sufficiently large negative values should be allowed to ensure bias-free reconstructions; $\psi=16$ was found to result in bias-free images under all circumstances. Note that the original NEGML method [13] implicitly used $\psi=1$ and that it was sometimes also used with even smaller values of $\psi$ (e.g. $10^{-4}$ in [11]) leading to remaining bias in the images.

Small values for $|A|$ in AML result in MLEM-like reconstruction properties, gradually increasing $|A|$ yields a transition to least squares reconstruction properties. When $|A|$ is sufficiently large, bias-free reconstructions are obtained. Note that in this work a fixed value for $A$ was chosen. Studying the influence of an nonuniform image $A_{j}$ with $j=1 . . J$, was beyond the scope of this paper.

For both algorithms large values for their parameter yield bias-free images. It turned out that least-squares-like behavior is required to obtain bias-free images. Both for NEGML and AML this bias-reduction comes at the cost of increased variance. No situation could be found where NEGML and AML have equal bias properties but significantly different variance behavior. Nevertheless, they both have lower variance than FBP, which is a useful property.

Parameters that give rise to bias-free NEGML and AML images often reduce the convergence rate of the hot region, even for relatively high counts. To reduce the required number of iterations ordered subsets can be used (to avoid limit cycle solutions the number of subsets could be decreased at higher iteration numbers). For NEGML, $\alpha_{j}$ could be chosen nonuniformly but care has to be taken since convergence will be slowed down for voxels with relatively low $\alpha_{j}$. We tested some choices where $\alpha_{j}$ increased monotonically with $\lambda_{j}$ (data not shown), the results were comparable to the results for small $|A|$ in AML: improved convergence in hot regions at the cost of risking more bias for cold regions.

This study also investigated the influence of the randoms handling on the bias in the image. The way the randoms are handled may have a large influence on the severeness of the bias. Ordinary Poisson with randoms smoothing copes best with noisy low count data. Ordinary Poisson without randoms smoothing shows the worst performance.

For precorrected data, the Shifted Poisson method as proposed by Yavuz and Fessler in [23] should be used instead of the Ordinary Poisson method. In a test using Shifted Poisson (data not shown), similar results have been obtained: the bias for Shifted Poisson without randoms smoothing was substantially increased compared to Shifted Poisson with randoms smoothing. The difference between Shifted Poisson and Ordinary Poisson was negligible compared to the difference between the use of unsmoothed and smoothed randoms. Randoms precorrection creates negative sinogram values, which must be set to zero in MLEM and therefore contribute a positive bias [5]. Since AML and NEGML can handle negative sinogram values, these algorithms do not encounter this problem. Under all circumstances both NEGML and AML (with large parameter) were able to mitigate the bias.

The experiments with the adjusted phantom 1 (threefold activity) and phantom 2 confirm the conclusions drawn for phantom 1, despite the fact that different activity and different 
projection settings were used. The experiments in this work have thus only been performed on two-dimensional data. However, a tenfold increase in the number of sinogram pixels for the same number of image pixels did not change the major results. Therefore, we anticipate that similar results will be obtained in 3D and time-of-flight PET, where the ratio of sinogram pixels to image pixels is also much larger.

\section{CONCLUSION}

Two algorithms are introduced that modify the Poisson likelihood in order to mitigate bias. Using very different mechanisms, both algorithms provide a kind of balance between the Poisson likelihood and the Gaussian likelihood (i.e. least squares), which is controlled by a single parameter.

NEGML combines a Poisson and Gaussian distribution and was shown to reduce the bias significantly when the parameter that determines the switch between both distributions, $\psi$, was at least equal to 16. AML is proposed as a simplification of ABML with upper bound $B$ set to infinity. It results in biasfree images when the lower bound $A$ is set low enough. Both algorithms are effective in reducing bias when their parameter was chosen high enough in magnitude. This comes at the cost of increased variance compared to MLEM but they both have lower variance than FBP.

Another issue that was studied was the influence of randoms handling. The use of unsmoothed randoms or randoms precorrected data increases the bias substantially. However, NEGML with $\psi \geq 16$ and AML with a "sufficiently high" magnitude of $A$ performed well for all randoms processing approaches.

Although the experiments in this work have been applied on two dimensional data, the results suggested that the conclusion drawn may hold for 3D and time-of-flight-PET configurations as well.

\section{REFERENCES}

[1] J. Qi and R. Leahy. "Iterative reconstruction techniques in emission computed tomography." Physics in Medicine and Biology, 51 (15), pp. R541-578, 2006.

[2] L.A. Shepp and V. Vardi. "Maximum likelihood reconstruction for emission tomography." IEEE Transactions Medical Imaging, 11(2), pp. $113-122,1982$

[3] K. Lange and R. Carson. "EM reconstruction for emission and transmission tomography." Journal of Computed Assisted Tomography, 8 (2), pp. 306-316, 1984

[4] R. Boellaard, A. van Lingen and A. A. Lammertsma. "Experimental and Clinical Evaluation of Iterative reconstruction (OSEM) in dynamic PET: quantitative characteristics and effects on kinetic modeling." Journal of Nuclear Medicine, 42 (5), pp. 808-817, 2001.

[5] F.H.P. van Velden, R. W. Kloet, B.N.M. van Berckel, S.P.A. Wolfensberger, A.A. Lammertsma and R. Boellaard. "Comparison of 3DOP-OSEM and 3D-FBP reconstruction algorithms for High-Resolution research tomograph studies: effects of randoms estimation methods. Physics in Medicine and Biology, 53, pp. 3217-3230, 2008.

[6] A. Reilhac, S. Tomeï, I. Buvat, C. Michel, F. Keheren and N. Costes. "Simulation-based evaluation of OSEM iterative methods in dynamic brain PET studies." NeuroImage, 39, pp. 359-268, 2008.

[7] H. Barrett and K. Myers. Foundations of Image Science. John Wiley and Sons, Hoboken/New Jersey, 2004

[8] C. Cloquet and M. Defrise. "MLEM and OSEM deviate from the Cramer-Rao bound at low counts." IEEE Transactions on Nuclear Science, 60 (1), pp. 134-143, 2013.

[9] C. Byrne. "Iterative algorithms for deblurring and deconvolution with constraints.", Inverse Problems 14, pp. 1455-1467, 1998.
[10] K. Erlandsson, D. Visvikis, W. Waddington, I. Cullum, P. Jarritt and L. Polowsky. "Low-statistics reconstruction with AB-EMML." Conference record IEEE Medical Imaging Conference, Lyon, 2, pp. 15/249-253, 2000

[11] J. Verhaeghe and A. Reader. "AB-OSEM reconstruction for improved Patlak kinetic parameter estimation: a simulation study." Physics in Medicine and Biology, 55, pp. 6739-6757, 2010.

[12] A. Rahmim, Y. Zhou, J. Tang, L. Lu, V. Sossi and D.F. Wong. "Direct 4D parametric imaging for linearized models of reversibly binding PET tracers using generalized AB-EM reconstruction." Physics in Medicine and Biology, 57, pp. 733-755, 2012.

[13] J. Nuyts, S. Stroobants, P. Dupont, S. Vleugels, P. Flamen and L. Mortelmans, "Reducing loss of image quality due to the attenuation artifact in uncorrected PET whole body images." Journal of Nuclear Medicine, 43, pp. 1054-1062, 2002.

[14] L. Grèzes-Besset, J. Nuyts, R. Boellaard, I. Buvat, C. Michel, C. Pierre, N. Costes and A. Reilhac. "Simulation-based evaluation of NEG-ML iterative reconstruction of low count PET data." Conference record IEEE Medical Imaging Conference, Hawaii, pp. 3009-3014, 2007.

[15] K. Van Slambrouck and J. Nuyts. "Reconstruction scheme for accelerated maximum likelihood reconstruction: the patchwork structure." (Submitted to IEEE Transactions on Nuclear Science: minor revision), 2013.

[16] K. Van Slambrouck and J. Nuyts. "Metal artifact reduction in computed tomography using local models in an image block-iterative scheme." Medical Physics, 39 (11), pp. 7080-7093, 2012.

[17] J. A. Fessler, E.P. Ficaro, N. H. Clinthorne and K. Lange , "Groupedcoordinate ascent algorithm for penalized-likelihood transmission image reconstruction.” IEEE Transactions on Medical Imaging, 16(2), pp. 166175,1997

[18] H. Hudson and R. Larkin. "Accelerated image reconstruction using ordered subsets of projection data.” Journal Theoretical Biology, 13(4), pp. $601-609,1994$.

[19] I. Hong, S. Cho, M. Casey and C. Michel. "Complementary reconstruction: improving image quality in dynamic PET studies." Conference record IEEE Medical Imaging Conference, Valencia, Spain, pp. 4327 4328, 2011.

[20] C. Watson. "Co-fan-sum ratio algorithm for randoms smoothing and detector normalization in PET". Conference record IEEE Medical Imaging Conference, Knoxville, TN, USA, pp. 3326-3329, 2010.

[21] C. Stearns, D. McDaniel, S. Kohlmeyer, P. Arul, B. Geiser and V. Shanmugan. "Random Coincidence Estimation from Single Event Rates on the Discovery ST PET/CT Scanner." Conference record IEEE Medical Imaging Conference, Portland, Oregan, USA, 5, pp. 3067-3069, 2003.

[22] S. Ahn and J. Fessler. "Emission image reconstruction for randomsprecorrected PET allowing negative sinogram values." IEEE Transactions Medical Imaging, 23(5), pp. 591-601, 2004.

[23] M. Yavuz and J. Fessler. "Statistical image reconstruction methods for randoms-precorrected PET scans." Medical Image Analysis, 2 (4), pp. 369-378, 1999. 